Antiviral drug discovery against arthritogenic alphaviruses: tools and molecular targets Rana Abdelnabi ${ }^{1 \#}$, Sofie Jacobs ${ }^{1 \#}$, Leen Delang $^{1 *}$, Johan Neyts $^{1 *}$

1. KU Leuven, Department of Microbiology, Immunology and Transplantation, Rega Institute for Medical Research, Laboratory of Virology and Chemotherapy, 3000 Leuven, Belgium.

\title{
"Equal contribution
}

*Corresponding authors:

Johan Neyts. Tel +3216337353, e-mail: Johan.Neyts@kuleuven.be.

Leen Delang. Tel +3216322107, e-mail: Leen.Delang@kuleuven.be. 


\section{Abstract}

Alphaviruses are (mainly) arthropod-borne viruses that belong to the family of the Togaviridae. Based on the disease they cause, alphaviruses are divided into an arthritogenic and an encephalitic group. Arthritogenic alphaviruses such as the chikungunya virus (CHIKV), the Ross River virus (RRV) and the Mayaro virus (MAYV) have become a serious public health concern in recent years. Epidemics are associated with high morbidity and the infections cause in many patients debilitating joint pain that can persist for months to years. The recent (20132014) introduction of CHIKV in the Americas resulted in millions of infected persons. Massive outbreaks of CHIKV and other arthritogenic alphaviruses are likely to occur in the future. Despite the worldwide (re-)emergence of these viruses, there are no antivirals or vaccines available for the treatment or prevention of infections with alphaviruses. It is therefore of utmost importance to develop antiviral strategies against these viruses. We here review the possible molecular targets in the replication cycle of these viruses for the development of antivirals. In addition, we provide an overview of the currently available in vitro systems and mouse infection models that can be used to assess the potential antiviral effect against these viruses.

Key words: Chikungunya; alphavirus; antivirals; mice; protease; replication 


\section{1. Burden of arthritogenic alphaviruses}

2 Alphaviruses (family Togaviridae) comprise a group of human and animal viruses, which are

3 mainly transmitted by the bite of hematophagous arthropods [1]. Based on their historical

4 geographical location, alphaviruses are classified into the New World and Old World

5 alphaviruses. The New World alphaviruses such as the Venezuelan and Western equine encephalitis viruses (VEEV and WEEV) are usually associated with encephalitic disease symptoms [1]. On the other hand, infections by the Old World alphaviruses e.g. chikungunya virus (CHIKV) and Ross river virus (RRV) result mainly in arthralgia and debilitating pain similar to pains caused by rheumatoid arthritis [1]. Acute infections by arthritogenic alphaviruses are self-limiting and mainly associated with fever, rash and arthritis [2]. However, many patients develop a chronic polyarthritis that can severely incapacitate the patient for weeks and even up to several years after the acute stage [2]. Recent CHIKV outbreaks have also been associated with severe complications such as Guillain-Barré syndrome [3] and meningoencephalitis [4].

Some arthritogenic alphaviruses are currently endemic in specific regions of the world, for example, O'nyong'nyong virus (ONNV) in Sub-Saharan Africa [5], RRV [6] and Barmah forest virus (BFV) [7] in Australia, and MAYV in the South and the Central Americas [8]. CHIKV on the other hand has re-emerged in several parts of the world in the last decade resulting in massive outbreaks that were associated with high morbidity rates [2]. At the end of 2013 , the first local transmission of CHIKV in the Americas has been reported on the Island of Saint Martin. Since then, millions of CHIKV infections have been reported in the Caribbean region and countries of Central and South America [2]. Potential expansion of infections with other arthritogenic alphaviruses are expected in the future. 
In general, arthritogenic alphaviruses are transmitted in enzootic/epizootic transmission cycles between an arthropod vector and animal reservoirs $[1,9]$. Transmission to humans typically occurs by direct spillover from these cycles via the bite of mosquitoes $[1,9]$. Until now, only CHIKV showed the ability for inter-human transmission, similar to dengue and Zika virus [9].

A wide variety of mosquito species has been reported to transmit arthritogenic alphaviruses. Aedes mosquitoes are the main vectors for CHIKV transmission [1]. ONNV is transmitted by Anopheles species [5]. For MAYV, Haemagogus species, mainly $H$. janthinomys, are the primary vectors for virus transmission [8]. However, other mosquitoes such as Ae. aegypti, Ae. albopictus [10] and four Anopheles species [11] have been reported to be competent vectors for MAYV in laboratory settings. On the other hand, RRV is transmitted by several mosquito species such as those belonging to the genera of Culex, Aedes, Anopheles and Mansonia [12]. Despite the worldwide (re-) emergence of CHIKV and other arthritogenic alphaviruses and their major clinical impact, there is no antiviral drug for the treatment or vaccine for the prevention of these viral infections. Therefore, the current treatment relies on symptomatic relief via the use of analgesics, antipyretics, non-steroidal anti-inflammatory drugs and, in severe cases, methotrexate [1]. Here, we review the potential molecular targets for inhibitors of arthritogenic alphaviruses replication (Fig 1). Moreover, we discuss the available in vitro and mouse models to assess the potential efficacy of such antiviral drugs.

\section{Genome organization of alphaviruses}

Alphaviruses are small (about 60-70 nm-diameter) positive-strand RNA viruses with an icosahedral-like nucleocapsid surrounded by a lipid envelope with embedded viral glycoproteins. The viral genome is approximately $12 \mathrm{~kb}$ and encodes two open reading frames (ORF), flanked by $5^{\prime}$ and $3^{\prime}$ untranslated regions with $5^{\prime}$ cap structures and a $3^{\prime}$ poly(A) tail [13]. 
The 5' ORF is translated from genomic RNA by a cap-dependent mechanism, which results in the formation of four non-structural proteins (nsP1-4) responsible for cytoplasmic RNA replication and modulation of cellular antiviral responses. The $3^{\prime}$ ORF is translated from a subgenomic RNA, which is also capped, to yield three major structural virus proteins (capsid, E2 and E1 envelope glycoproteins) and two small peptides (6k and E3) [13].

\section{Viral targets for anti-alphavirus compounds}

\subsection{Viral entry}

Alphaviruses enter the host cell by receptor-mediated endocytosis following the interaction of the viral E2 glycoprotein with specific cellular receptors (Fig 2) [14]. Within the endosome, conformational changes of the viral envelope glycoprotein E1 are triggered by the low $\mathrm{pH}$ leading to its fusion with the endosomal membrane (Fig 2) [14]. Consequently, the viral nucleocapsid is released into the cytoplasm where it disassembles to release the viral RNA genome [14]. Molecules that raise the endosomal $\mathrm{pH}$, such as the antimalarial drug chloroquine $[15,16]$ and the anticancer drug obatoclax [17], have therefore been shown to inhibit the in vitro replication of alphaviruses (including CHIKV and SFV) through preventing the viral fusion step. Disruption of the alphavirus envelope structure by amphipathic molecules such as porphyrins was also shown to inhibit the binding and entry of CHIKV, MAYV and Sindbis virus (SINV) [18]. Another strategy to inhibit viral entry is the use of molecules that target alphavirus-specific receptors. For example, flavaglines are natural products that were reported to target prohibitin-1, a signaling protein that was identified as a receptor for $\mathrm{CHIKV}$ in mammalian cells [19]. Two synthetic flavaglines derivatives were shown to inhibit CHIKV replication in cell culture and to reduce the co-localization of prohibitin-1 and the CHIKV E2 glycoprotein which may suggest an effect on CHIKV binding to this receptor [20]. Arbidol is a broad-spectrum antiviral that has been reported to be an early stage inhibitor of CHIKV 
replication in cell cultures [21]. An arbidol-resistant variant was identified that carries a glycine to an arginine (G407R) mutation in the CHIKV E2 glycoprotein, which is the protein involved in the viral binding to host receptors [21]. Recent studies report that the anti-trypanosomiasis drug suramin has antiviral activity against different CHIKV isolates and related alphaviruses in vitro [22,23]. In addition, suramin reduced CHIKV-induced arthritis in CHIKV-infected C57BL/6 mice [24]. Suramin inhibits CHIKV entry and cell-to-cell transmission, most probably through binding to the cavity between CHIKV glycoprotein E1 domain II and E2 domain C [22,23]. Several human and murine monoclonal antibodies (mAbs, reviewed in [25]) inhibit the entry of CHIKV and other arthritogenic alphaviruses. The mechanism of viral entry inhibition by such mAbs is mainly via interference with receptor attachment or via blocking of post-attachment steps required for the viral membrane fusion [25]. Recently, the adhesion molecule Mxra8 has been identified as an entry receptor for several arthritogenic alphaviruses including CHIKV, ONNV, RRV and MAYV [26]. An Mxra8-Fc fusion protein and anti-Mxra8 mAbs inhibit CHIKV infection in various cell types and were also shown to be able to reduce CHIKV and ONNV infection in C57BL/6 mice [26].

\subsection{Viral RNA capping}

The mechanism by which the alphavirus non-structural protein 1 (nsP1) is responsible for the capping of the viral RNA is distinct from the conventional capping mechanism of the host cell. A methylgroup transfer and subsequent formation of an $\mathrm{m}^{7} \mathrm{GMP}-\mathrm{nsP} 1$ covalent complex is mediated by the methyl- and guanylyltransferase activities of nsP1. In a final nsP1 mediated reaction, $\mathrm{m}^{7} \mathrm{GMP}$ is attached to the $5^{\prime}$ end of the viral $\mathrm{RNA}$, resulting in the formation of a cap0 structure [27]. In addition, it has been suggested that interaction of nsP1 with all other nsPs is essential to keep the replication complexes intact and functional [28]. These observations make nsP1 an attractive antiviral drug target [27]. The first class of small molecules reported 
to target nsP1 (the MADTP-series) were shown to be active also against clinical CHIKV isolates in cell culture. This class of molecules inhibits the guanylyltransferase activity of enzymatically active VEEV nsP1 in vitro [29-31]. The guanosine analog, ribavirin, depletes intracellular GTP pools, which prevents proper capping of newly synthesized viral RNA by nsP1 [32]. Consequently, this may impede viral RNA translation and allow host $5^{\prime}$ exonucleases to degrade uncapped viral RNA [28]. More recently, the naturally derived compound lobaric acid was identified in a high-throughput screen as an inhibitor of CHIKV nsP1 GTP binding and guanylation [33]. Antiviral efficacy of lobaric acid was demonstrated in cell-based antiviral assays against both SINV and CHIKV [33].

\subsection{Viral protease activity}

The non-structural protein 2 (nsP2) of alphaviruses is a multifunctional protein that encodes RNA helicase, nucleoside triphosphatase and RNA triphosphatase activities within its Nterminal. It possesses also a cysteine protease activity at its C-terminal that is involved in cleavage of the non-structural viral polyprotein (Fig 2) [28]. nsP2 has also been reported to be involved in shutting off host cell mRNA transcription and translation and to inhibit cellular antiviral response (shut off of the JAK-STAT signaling pathway) [28]. Targeting the viral protease has been shown to be a promising and a powerful strategy to inhibit viral replication in patients infected with either HIV or HCV [34]. It is thus conceivable that the nsP2 protease of alphaviruses could be also a promising target for drug discovery and development.

The crystal structures or homology models of the nsP2 protease of different arthritogenic alphaviruses were explored in an attempt to identify potential inhibitors through a combination of molecular docking and molecular dynamics studies [35-37]. Based on this approach, some hit compounds and five potential binding pockets of the CHIKV nsP2 protease were identified [35]. Some arylalkylidene derivatives of 1,3-thiazolidin-4-one that inhibit the 
in vitro $\mathrm{CHIKV}$ replication ( $\mathrm{EC}_{50}$ values in the low $\mu \mathrm{M}$ range) have been proposed, based on

121 molecular docking studies, to target the nsP2 protease domain [38]. A panel of in silico

122 predicted inhibitors of the CHIKV protease [37] as well as some peptidomimetics [39] were

123 confirmed to inhibit the CHIKV nsP2 protease activity and were shown to also inhibit the viral

124 replication in cell culture.

125

126

127

\subsection{Viral genome replication}

For viruses such as HIV, HBV, HCV, influenza and herpes, the viral polymerase has shown to be an excellent target for antivirals. The alphavirus nsP4, functions as the viral RNA-dependent RNA polymerase (RdRp) [14], may thus be considered one of the most attractive targets for the development of (broad-spectrum) anti-alphavirus compounds. The anti-influenza drug favipiravir and its defluorinated analog, $\mathrm{T}-1105$, have been proven to inhibit the replication of CHIKV and related (arthritogenic) alphaviruses in cell culture [40]. Favipiravir treatment (300 $\mathrm{mg} / \mathrm{kg} /$ day for 7 days) of CHIKV-infected AG129 mice protected from severe neurological disease and markedly increased the survival rate [40]. Furthermore, treating CHIKV-infected C57BL/6J mice with favipiravir (300 mg/kg/day for 4 days) reduced viral replication in the joints of the extremities during the acute phase of infection [41].

\section{Targeting host factors}

\subsection{Intracellular nucleotide depletion}

Ribavirin (a guanosine analogue) and mycophenolic acid have been reported to inhibit CHIKV replication. Both molecules inhibit the inosine monophosphate dehydrogenase enzyme (IMPDH) resulting in the depletion of GTP pools $[42,43]$. 6-Azauridine, which inhibits orotidine monophosphate decarboxylase enzyme (OMP) resulting in depletion of the intracellular UTP pools, inhibits the in vitro replication of CHIKV and SFV [44]. However, the cytotoxicity of this 
143 compound increased in exponentially growing cells and should thus be considered when

144 interpreting the antiviral efficacy.

\section{$145 \quad$ 4.2. Protein synthesis machinery}

146 Some molecules have been shown to inhibit alphavirus replication by interfering with the

147 translation of viral proteins via aspecific mechanisms. Harringtonine, an inhibitor of eukaryotic 148 protein synthesis, was shown to inhibit the in vitro replication of CHIKV (including in primary

149 human skeletal myoblasts) and SINV [45].

\section{$150 \quad$ 4.3. Cellular chloride channels}

151 Cellular ion channels has been proven to play an important role during the entry and 152 replication of several viruses [46]. Recently, two chloride channels (CLIC1 and CLIC4) have 153 been identified as pro-viral factors for CHIKV replication using siRNA-mediated knock down 154 [47]. The chloride channel inhibitors diisothiocyanostilbene-2,20-disulfonic acid (DIDS), 9anthracene carboxylic acid (9-ACA) and 5-nitro-2-3-phenylpropylamino benzoic acid (NPPB)

156 result in in vitro antiviral activity against CHIKV in Huh7 cells [47]. NPPB also inhibited CHIKV 157 replication in mosquito (C6/36) cells [47].

\section{4.4. Cellular furins}

159 During alphavirus replication, the viral glycoprotein precursor is processed by cellular furins

160 to produce mature virions (Fig 2). Treating CHIKV-infected human muscle satellite cells with the furin inhibitor decanoyl-RVKR-chloromethyl ketone (dec-RVKR-cmk) has been reported to inhibit viral infection [48]. As a result of furin inhibition by dec-RVKR-cmk, immature viral particles were produced and in turn the viral spreading was markedly reduced [48].

\subsection{Cellular kinases}

165 Some cellular kinases have been identified to play a key role during arthritogenic alphavirus replication. The Src family kinases (SFKs) are a family of membrane-associated kinases that 
mediate signal transduction of several receptors and that have been reported to promote the replication of various viruses such as hepatitis C virus (HCV) and dengue virus (DENV) [49]. A Kinome study was performed, in CHIKV-infected human dermal fibroblasts, to identify which kinase pathways are significantly altered as a result of CHIKV infection [49]. It was shown that the SFK-phosphatidylinositol 3-kinase (PI3K)-AKT-mTORC-signaling pathway is activated in CHIKV infected cells. Treating human fibroblasts with the SFK inhibitor (dasatinib) or the mTORC1/2 inhibitor (Torin 1) reduced the virus yield of CHIKV, ONNV, RRV and MAYV [49]. This antiviral effect was mediated by inhibition of the alphavirus subgenomic RNA translation and hence inhibition of the structural protein synthesis. Also the major mitogen-activated protein kinase (MAPK) signaling pathways are activated during CHIKV infection and play a role in infectious alphavirus particles formation [50]. Reducing the MAPK pathway activation by berberine, a plant-derived alkaloid, was reported to inhibit the in vitro replication of ONNV and different CHIKV isolates. Furthermore, berberine treatment significantly reduced the joint swelling and inflammation at its peak (i.e. day 6 post-infection) in CHIKV-infected mice [50].

Activation of the protein kinases C (PKCs) by phorbol esters e.g. prostratin [51] or salicylatebased bryostatin analogs [52] has been reported to inhibit the in vitro replication of CHIKV. Unlike phorbol esters, bryostatin has no tumor-promoting effect. Interestingly, byrostatinbased analogs with a cap in their scaffold that inhibit or reduce binding to PKCs were still able to inhibit CHIKV replication [52]. Combination with different PKC inhibitors counteracted the antiviral activity of a non-capped analog but did not affect that of capped analogs, which further confirmed that the capped analogs inhibit CHIKV replication via a PKC-independent mechanism [52].

\subsection{Host immune response}


A recent study reported on elucidating the subtype-specific mechanisms by which IFN- $\alpha$ and

191 IFN- $\beta$ exert a protective role during acute alphavirus infection, highlighting their importance 192 in controlling the infection [53]. We here discuss how the possibility of treating arthritogenic 193 alphavirus disease by targeting the host immune response has been explored using different 194 strategies. Recombinant IFN- $\alpha$ inhibits the replication of CHIKV and SFV in vitro [44]. However, 195 suppressing CHIKV production with IFN- $\alpha$ monotherapy requires concentrations exceeding feasible clinical treatment regimens [54]. Mathematical modelling was used to investigate the potential of ribavirin and IFN- $\alpha$ combination therapy against CHIKV. The simulation outputs were validated experimentally showing a 99\% reduction in CHIKV levels by combining ribavirin and IFN- $\alpha$ at standard clinical regimens [54]. Type I IFN associated factors have also been considered as potential therapeutics. For example, viperin, an IFN-inducible protein has been

201 reported to inhibit a broad spectrum of DNA and RNA viruses [55]. Evidence of its antiviral 202 function was further supported by in vivo data from a study involving SINV [56]. Another 203 example is polyinosinic acid:polycytidylic acid (poly $(\mathrm{l}: \mathrm{C})$ ). This synthetic analogue of dsRNA has 204 been shown to upregulate the toll-like receptor-3 (TLR3) which results in the induction of IFN$205 \alpha / \beta$ and other antiviral genes (e.g. OAS and MxA) in mice $[57,58]$. Activation of retinoic acid 206 inducible gene-I (RIG-I) by viral nucleic acids results in downstream signaling leading to 207 increased levels of type I IFNs [59]. Previous studies have described the therapeutic potential 208 of RIG-I agonists as antiviral agents against different viruses including alphaviruses, both in 209 vitro and in vivo [60,61]. Recently, a small-molecule agonist of the adaptor protein STING was suggested to be a novel drug candidate due to its ability to produce an antiviral type I IFN 211 response [62]. In this study, the molecule C11 was shown to elicit conditions in human 212 telomerase-transduced foreskin fibroblast cells that are refractory to the growth of multiple 213 alphaviruses [62]. 
214 Heparan sulfate mimetics which can modulate the levels of inflammatory infiltrates and

215 cytokines, were found to reduce the severity of alphavirus-induced pathologies in mice $216[63,64]$. Particularly, pentosan polysulfate is currently undergoing phase II clinical trials 217 (PARA_004, Paradigm BioPharmaceuticals) for patients diagnosed with RRV-induced arthritic 218 disease [64].

\section{In vitro and in vivo infection models}

$220 \quad$ 5.1. Viruses

221 Surrogate and bio-safe alphaviruses

222 Since some arthritogenic alphaviruses, such as CHIKV, require high biosafety level facilities to 223 carry out antiviral studies, several surrogate systems have been developed to allow 224 conducting such studies at a lower biosafety level. One example is the use of an attenuated 225 strain of the virus e.g. CHIKV vaccine strain 181/25 [49]. Another example is to use stable 226 mammalian cell lines that contain a persistently replicating CHIKV replicon which allow to 227 screen for replication inhibitors $[65,66]$. To assess whether certain molecules act as entry 228 inhibitors, pseudo-viral particles that carry the alphavirus envelope proteins and express a 229 luciferase reporter can be used $[67,68]$. In addition, arthritogenic alphaviruses with a lower 230 biosafety level such as the Semliki Forest virus (SFV) are of value for initial screening for 231 alphavirus inhibitors, hits can be then profiled against alphaviruses of a higher biosafety level $232[65]$.

\section{Pathogenic alphaviruses}

234 Various laboratory strains and clinical isolates of arthritogenic alphaviruses have been used to 235 evaluate the efficacy of antiviral compounds. For CHIKV, the laboratory adapted strains 236 include ROSS [69,70], LR2006_OPY1 (GenBank: DQ443544.2) [38], CHIKV-S27 (ATCC: VR-64, 237 African prototype)[23] and CHIKV 899 (GenBank: FJ959103.1, an Indian Ocean strain) [40]. 
238 Clinical CHIKV isolates have also been used for antiviral screening purpose such as CHIKV-0708

239 (GenBank: FJ513654) [69,71], DRDE-06 (GenBank: EF210157) [42], Venturini and Bianchi and

240 Congo 95 [31]. In addition, CHIKV isolates that carry the mosquito adapting A226V mutation

241 in the E1 protein [e.g. CHIKV-122508 (GenBank: FJ445502.2) and the 0810bTw (GenBank:

242 FJ807899)] are available [23,71,72].

243 Unlike for CHIKV, antiviral studies for the other arthritogenic alphaviruses are sparse.

244 Examples for available strains of the other arthritogenic alphaviruses include: MAYV (ATCC VR 66, strain TR4675) [73], MAYV BeAr20290 strain (GenBank: KY618127) [74], ONNV strain IPD

247 (GenBank: GQ433359, a laboratory strain) [64]. Convenient tools for high-throughput

249 fluorescence protein or a luciferase gene such as the green-fluorescent protein (GFP) [75], 250 Renilla luciferase (Rluc) [65] or Gaussia luciferase (Gluc) [50].

\section{5.2. Cell culture models}

252 Cell-based antiviral assays that rely on the infection of susceptible cells with a virus are 253 commonly used in the initial stage of alphavirus drug discovery $[14,76,77]$. The antiviral 254 efficacy can be expressed as a reduction in virus-induced cytopathic effect (CPE), which can 255 be scored microscopically and/or quantified using colorimetric assays $[78,79]$. Alternatively, 256 when the cell type is not susceptible to the virus-induced CPE, the antiviral efficacy can be 257 expressed as a reduction in fluorescence or luminescence using specific reporter viruses [76]. 258 Arthritogenic alphaviruses infect a wide range of cell types and tissues, including monocytes 259 and/or macrophages, dendritic cells, synovial and dermal fibroblasts, endothelial cells and 260 muscle cells [80]. However, other cell types are most commonly used for antiviral screening, 261 including African green monkey kidney (Vero) cells, baby hamster kidney (BHK) cells, HeLa 
cells, human fetal lung fibroblast (MRC-5) cells and human embryonic kidney 293 (HEK-293T)

263 cells $[76,77,81]$. Although these cells have little to no clinical relevance to alphavirus 264 infections, they are very suitable for high-throughput screening campaigns. Recently, four 265 mammalian cells lines; Huh7 (hepatocyte, human), C2C12 (myoblast, mouse), SVG-A 266 (astroglia, human) and dermal fibroblasts (transformed cell line, human); were proposed as 267 good cell culture models for in vitro CHIKV research [82]. These cell lines are biologically and 268 clinically relevant in the context of arthritogenic alphavirus infections. Two of these proposed 269 cell lines were previously used in high-throughput screening applications $[83,84]$. These 270 reports implicate the potential of using clinically relevant cell lines in future drug discovery 271 campaigns for alphaviruses.

\section{$272 \quad$ 5.3. Mouse models}

273 Mouse models are commonly used to preclinically assess the efficacy of antiviral drug 274 candidates in vivo. Both immunocompetent and immunocompromised mouse models are 275 available to evaluate the efficacy of antivirals against arthritogenic alphaviruses. Using these 276 models, the antiviral efficacy can be assessed based on quantification of infectious virus titers 277 in serum and target organs, histopathology and clinical disease scores. Adult C57BL/6 mice are 278 the main immunocompetent model for arthritogenic alphavirus infections [85-90]. For some 279 alphaviruses, newborn and adult Swiss, adult CD-1 and BALB/c mice have been used as 280 alternative immunocompetent models $[87,89,91,92]$. To recapitulate the pathologies 281 associated with acute human infections, the mice are inoculated subcutaneously at the ventral 282 side of the rear foot pad $[93,94]$. On day 6-8 post infection, animals display peak swelling of 283 the inoculated foot and occur with signs of moderate arthritic disease defined by hunching, 284 lethargy and gait alteration. Infectious virus can be detected in serum, skeletal muscle and 285 distant joint tissues such as ankles and wrists. Signs of joint and skeletal muscle inflammation 
can be observed after histopathological examination. Infection of C57BL/6 mice with SFV or

SINV also leads to viral replication in the central nervous system, resulting in paralysis and

288 death [95-97]. Thus, this particular model is mainly used to study the effect on alphavirus

289 neurological pathogenesis. A limited number of immunocompromised mouse models are

290 available to study CHIKV and the closely related viruses, ONNV and MAYV [40,90,94]. The mice

291 used in these models are deficient in type I or type I and type II interferon pathways. Infection

292 of these mice results in viral replication in skeletal muscle, myositis and high mortality, which

293 can be used as end-point markers for testing the antiviral efficacy.

\section{6. Future directions}

295 The worldwide re-emergence of arthritogenic alphaviruses and the high morbidity rate 296 associated with their infections make these viruses an emerging health threat. The 297 development of potent and safe antiviral compounds against arthritogenic alphaviruses is 298 therefore urgently needed. Such antivirals will help to reduce the severity of the disease 299 symptoms during the acute phase of infection. In addition, reduction of the viral load in 300 infected patients by antiviral treatment may help to decrease the transmission efficiency of 301 the virus by mosquitoes [98]. Since patients with more severe symptoms during the acute 302 phase of alphavirus infection have a higher chance to develop chronic joint disease [99], the 303 use of a potent antiviral during the acute infection is expected to decrease the likelihood to 304 develop chronic symptoms. Household-prophylaxis may also be a potential application of 305 antivirals as the probability of arthritogenic alphavirus transmission such as CHIKV was shown 306 to be up to $12 \%$ between household members [100].

307 To date, highly potent drugs are only available for the treatment of a limited number of viruses 308 such as HIV, HBV, HCV, influenza and herpes viruses. However, with sufficient time and effort, 309 it should also be possible to develop safe and potent antivirals for the treatment and/or 
prophylaxis of arthritogenic alphavirus infections. Potential targets for development of broad-

311 spectrum antivirals for arthritogenic alphaviruses may for example be the viral nsP1 (capping

312 machinery), nsP2 (protease) and nsP4 (viral polymerase) proteins. Another potential target is

313 Mxra8 which functions as an entry receptor for several arthritogenic alphaviruses [26].

314 Designing molecules that can block this receptor may be therefore a helpful strategy to control 315 arthritogenic alphavirus infections. 


\section{Acknowledgments}

318 Funding provided by Fund for Scientific Research (FWO) Flanders individual credit (1522918N)

319 for L.D. S. J. was supported by FWO PhD fellowship (1S21918N).

320 Conflict of interest

321 None to declare. 
<smiles>CCOCOc1ccc(NC(C)CCCN(CC)CC)c2ccc(Cl)cc12</smiles><smiles>COC(=O)C[C@](O)(CCC(C)(C)O)C(=O)O[C@H]1C(OC)=C[C@]23CCCN2CCc2cc4c(cc2[C@H]13)OCO4</smiles>

Harringtonine<smiles>COC1=CC(c2cc3ccccc3[nH]2)=N/C1=C\c1[nH]c(C)cc1C</smiles>

Obatoclax<smiles>NC(=O)c1ncn(C2OC(CO)C(O)C2O)n1</smiles>

Ribavirin<smiles>CCOC(=O)c1c(CSc2ccccc2)n(C)c2cc(Br)c(O)c(CN(C)C)c12</smiles>

Arbidol<smiles>NC(=O)c1nc(F)cnc1O</smiles>

Favipiravir(T-705)
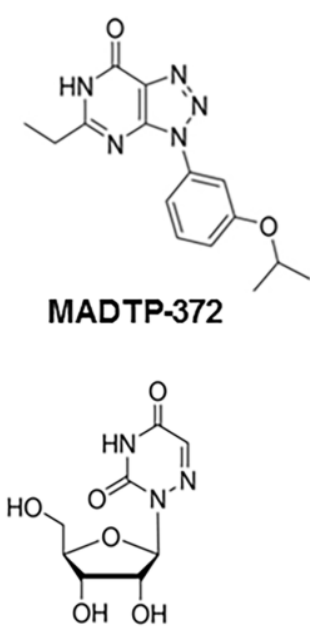

6-azuridine<smiles>Cc1nc(Nc2ncc(C(=O)Nc3c(C)cccc3Cl)s2)cc(N2CCN(CCO)CC2)n1</smiles>

Dasatinib<smiles>COc1ccc2cc3[n+](cc2c1OC)CCc1cc2c(cc1-3)OCO2</smiles>

Berberine<smiles>CCCCCCCCCC(=O)NC(CCCNC(=N)N)C(=O)NC(C(=O)NC(CCCCN)C(=O)NC(CCCNC(=N)N)C(=O)CCl)C(C)C</smiles>
dec-RVKR-cmk

324 Figure 1. Structural formulae of molecules inhibiting arthritogenic alphaviruses. 


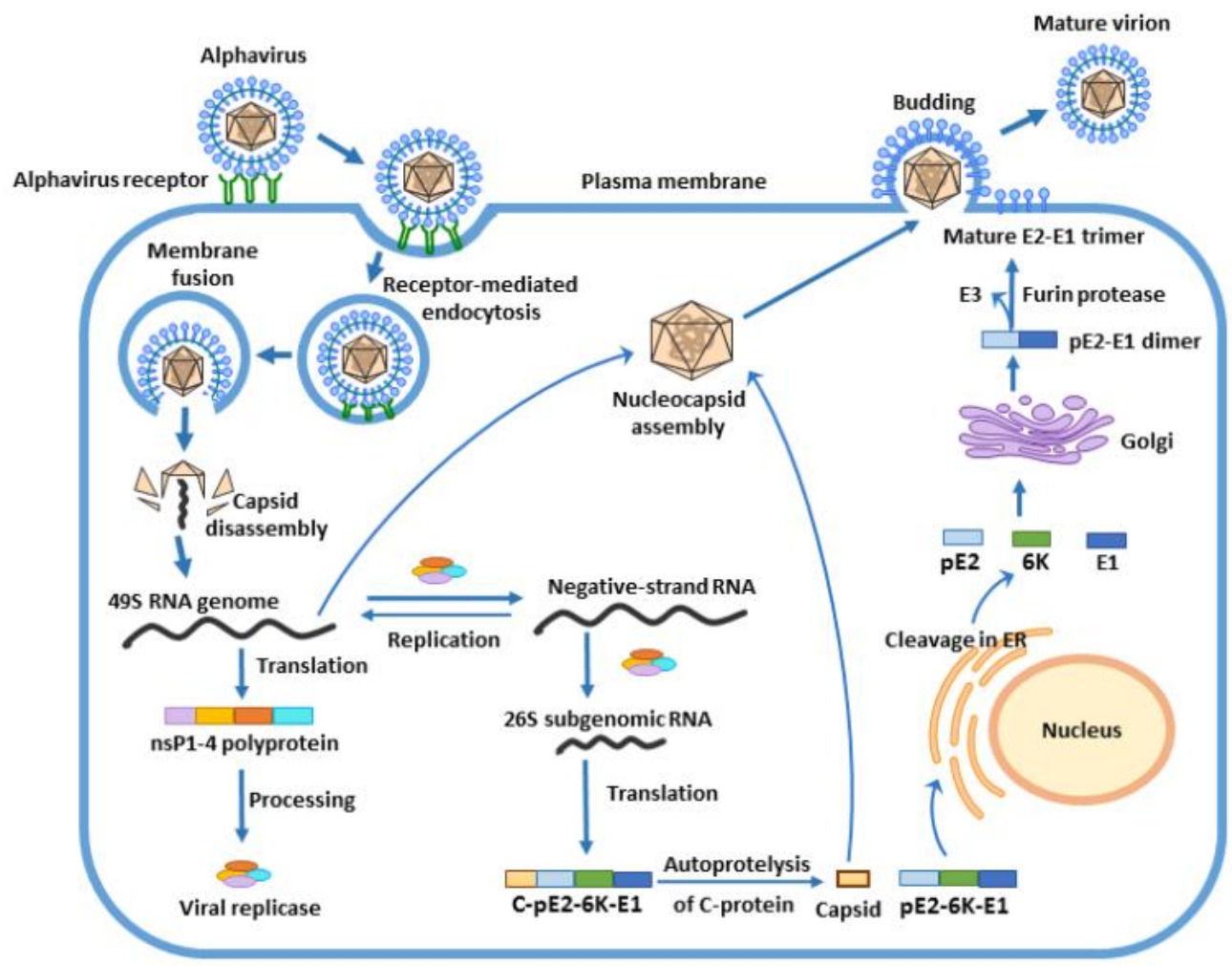

327 Figure 2. Schematic representation of the replication cycle of alphaviruses. Alphavirus enters

328 the cell by endocytosis following the binding of E2 protein to specific receptor(s) on the cell

329 surface. Within the endosome, the low $\mathrm{pH}$ triggers the fusion of the viral envelope with the endosomal membrane leading to the release of the nucleocapsid into the cytoplasm. The nucleocapsid disassembles to liberate the viral genome which is being translated to produce the viral nonstructural proteins (nsP1-4). After processing, the viral nonstructural proteins complex to form the viral replicase which catalyzes the synthesis of a negative-sense RNA

334 strand to serve as a template for synthesis of both the full-length positive-sense genome and 335 the subgenomic (26S) RNA. The subgenomic (26S) RNA is being translated to produce the 336 structural polyprotein (C-E3-E2-6K-E1). The structural polyprotein is then cleaved to produce 337 the individual structural proteins followed by assembly of the viral components. The 338 assembled virus particle is released by budding out through the plasma membrane where it 339 acquires the envelope with embedded viral glycoproteins. The figure was adapted from [14]. 


\section{References}

342 [1] L.I. Levi, M. Vignuzzi, Arthritogenic Alphaviruses: A Worldwide Emerging Threat?, Microorganisms. 7 (2019) 133. doi:10.3390/microorganisms7050133.

344 [2] G. Rezza, S.C. Weaver, Chikungunya as a paradigm for emerging viral diseases: Evaluating disease impact and hurdles to vaccine development, PLoS Negl. Trop. Dis. 13 (2019) 1-12. doi:10.1371/journal.pntd.0006919.

[3] E. Oehler, E. Fournier, I. Leparc-Goffart, P. Larre, S. Cubizolle, C. Sookhareea, S. Lastère,

F. Ghawche, Increase in cases of guillain-barré syndrome during a chikungunya outbreak, French Polynesia, 2014 to 2015, Eurosurveillance. 20 (2015). doi:10.2807/1560-7917.ES.2015.20.48.30079.

[4] P. Gérardin, T. Couderc, M. Bintner, P. Tournebize, M. Renouil, J. Lémant, V. Boisson, G. Borgherini, F. Staikowsky, F. Schramm, M. Lecuit, A. Michault, Encephalchik Study Group, Chikungunya virus-associated encephalitis: A cohort study on La Réunion Island, 2005-2009., Neurology. 86 (2016) 94-102. doi:10.1212/WNL.0000000000002234.

[5] G. Rezza, R. Chen, S.C. Weaver, O’nyong-nyong fever: a neglected mosquito-borne viral disease, Pathog. Glob. Health. 111 (2017) $271-275$. doi:10.1080/20477724.2017.1355431.

[6] X. Liu, K. Tharmarajah, A. Taylor, Ross River virus disease clinical presentation, 359 pathogenesis and current therapeutic strategies, Microbes Infect. 19 (2017) 496-504. doi:10.1016/j.micinf.2017.07.001.

361 [7] N. Kurucz, P. Markey, A. Draper, L. Melville, R. Weir, S. Davis, A. Warchot, R. Boyd, D. Stokeld, Investigation into High Barmah Forest Virus Disease Case Numbers Reported in the Northern Territory, Australia in 2012-2013, Vector-Borne Zoonotic Dis. 16 (2016) 
[8] Y. Acosta-Ampudia, D.M. Monsalve, Y. Rodríguez, Y. Pacheco, J.M. Anaya, C. RamírezSantana, Mayaro: an emerging viral threat?, Emerg. Microbes Infect. 7 (2018) 1-11. doi:10.1038/s41426-018-0163-5.

[9] S.C. Weaver, Prediction and prevention of urban arbovirus epidemics: A challenge for the global virology community, Antiviral Res. 156 (2018) 80-84. doi:10.1016/j.antiviral.2018.06.009.

[10] K. Wiggins, B. Eastmond, B.W. Alto, Transmission potential of Mayaro virus in Florida Aedes aegypti and Aedes albopictus mosquitoes, Med. Vet. Entomol. 32 (2018) 436442. doi:10.1111/mve.12322.

[11] M. Brustolin, S. Pujhari, C.A. Henderson, J.L. Rasgon, Anopheles mosquitoes are competent MAYV vectors Anopheles Mosquitoes May Drive Invasion and Transmission of Mayaro Virus across, (2018) 1-11. doi:10.1101/359349.

[12] S.B. Claflin, C.E. Webb, Ross River Virus: Many Vectors and Unusual Hosts Make for an Unpredictable Pathogen, PLOS Pathog. 11 (2015) e1005070. doi:10.1371/journal.ppat.1005070.

[13] J. Jose, A.B. Taylor, R.J. Kuhn, Spatial and Temporal Analysis of Alphavirus Replication and Assembly in Mammalian and Mosquito Cells, MBio. 8 (2017). doi:10.1128/mbio.02294-16.

[14] R. Abdelnabi, J. Neyts, L. Delang, Towards antivirals against chikungunya virus, Antiviral Res. 121 (2015) 59-68. doi:10.1016/j.antiviral.2015.06.017.

[15] E. Bernard, M. Solignat, B. Gay, N. Chazal, S. Higgs, C. Devaux, L. Briant, Endocytosis of chikungunya virus into mammalian cells: Role of clathrin and early endosomal compartments, PLoS One. 5 (2010). doi:10.1371/journal.pone.0011479.

[16] M. Khan, S.R. Santhosh, M. Tiwari, P. V. Lakshmana Rao, M. Parida, Assessment of in 
vitro prophylactic and therapeutic efficacy of chloroquine against Chikungunya virus in Vero cells, J. Med. Virol. 82 (2010) 817-824. doi:10.1002/jmv.21663.

[17] A.T. Varghese FS, Rausalu K, Hakanen M, Saul S, Kümmerer BM, Susi P, Merits A, 392 Obatoclax inhibits alphavirus membrane fusion by neutralizing the acidic environment of endocytic compartments., Antimicrob Agents Chemother. (2016) AAC.02227-16.

[18] R.L.S. Neris, C.M. Figueiredo, L.M. Higa, D.F. Araujo, C.A.M. Carvalho, B.R.F. Verçoza, 395

[20] P. Wintachai, F. Thuaud, C. Basmadjian, S. Roytrakul, S. Ubol, L. Désaubry, D.R. Smith, Assessment of flavaglines as potential chikungunya virus entry inhibitors, Microbiol. Immunol. 59 (2015) 129-141. doi:10.1111/1348-0421.12230.

[21] I. Delogu, B. Pastorino, C. Baronti, A. Nougairède, E. Bonnet, X. de Lamballerie, In vitro antiviral activity of arbidol against Chikungunya virus and characteristics of a selected resistant mutant, Antiviral Res. 90 (2011) 99-107. doi:10.1016/j.antiviral.2011.03.182.

[22] I.C. Albulescu, M. Van Hoolwerff, L.A. Wolters, E. Bottaro, C. Nastruzzi, S.C. Yang, S.C. Tsay, J.R. Hwu, E.J. Snijder, M.J. Van Hemert, Suramin inhibits chikungunya virus replication through multiple mechanisms, Antiviral Res. 121 (2015) 39-46. doi:10.1016/j.antiviral.2015.06.013. 
413 [23] Y.J. Ho, Y.M. Wang, J.W. Lu, T.Y. Wu, L.I. Lin, S.C. Kuo, C.C. Lin, Suramin inhibits

414

415

416

417

418

419

420

421

422

423

424

425

426

427

428

429

430

431

432

433

434

435

436

chikungunya virus entry and transmission, PLoS One. 10 (2015). doi:10.1371/journal.pone.0133511.

[24] S.-C. Kuo, Y.-M. Wang, Y.-J. Ho, T.-Y. Chang, Z.-Z. Lai, P.-Y. Tsui, T.-Y. Wu, C.-C. Lin, Suramin treatment reduces chikungunya pathogenesis in mice, Antiviral Res. 134 (2016) 89-96. doi:10.1016/j.antiviral.2016.07.025.

[25] J. Jin, G. Simmons, Antiviral Functions of Monoclonal Antibodies against Chikungunya Virus, Viruses. 11 (2019) 305. doi:10.3390/v11040305.

[26] R. Zhang, A.S. Kim, J.M. Fox, S. Nair, K. Basore, W.B. Klimstra, R. Rimkunas, R.H. Fong, H. Lin, S. Poddar, J.E. Crowe, B.J. Doranz, D.H. Fremont, M.S. Diamond, Mxra8 is a receptor for multiple arthritogenic alphaviruses, Nature. 557 (2018) 570-574. doi:10.1038/s41586-018-0121-3.

[27] R. Kaur, R. Mudgal, M. Narwal, S. Tomar, Development of an ELISA assay for screening inhibitors against divalent metal ion dependent alphavirus capping enzyme, Virus Res. 256 (2018) 209-218. doi:10.1016/J.VIRUSRES.2018.06.013.

[28] F. Abu Bakar, L.F.P. Ng, Nonstructural Proteins of Alphavirus-Potential Targets for Drug Development., Viruses. 10 (2018). doi:10.3390/v10020071.

[29] C. Li, J. Guillén, N. Rabah, A. Blanjoie, F. Debart, J.-J. Vasseur, B. Canard, E. Decroly, B. Coutard, mRNA Capping by Venezuelan Equine Encephalitis Virus nsP1: Functional Characterization and Implications for Antiviral Research, J. Virol. 89 (2015) 8292-8303. doi:10.1128/JVI.00599-15.

[30] A. Gigante, A. Gómez-SanJuan, L. Delang, C. Li, O. Bueno, A.-M. Gamo, E.-M. Priego, M.J. Camarasa, D. Jochmans, P. Leyssen, E. Decroly, B. Coutard, G. Querat, J. Neyts, M.-J. Pérez-Pérez, Antiviral activity of [1,2,3]triazolo[4,5-d]pyrimidin-7(6H)-ones against 
chikungunya virus targeting the viral capping nsP1, Antiviral Res. 144 (2017) 216-222. doi:10.1016/J.ANTIVIRAL.2017.06.003.

[31] L. Delang, C. Li, A. Tas, G. Quérat, I.C. Albulescu, T. De Burghgraeve, N.A. Segura Guerrero, A. Gigante, G. Piorkowski, E. Decroly, D. Jochmans, B. Canard, E.J. Snijder, M.J. Pérez-Pérez, M.J. Van Hemert, B. Coutard, P. Leyssen, J. Neyts, The viral capping enzyme nsP1: A novel target for the inhibition of chikungunya virus infection, Sci. Rep. 6 (2016). doi:10.1038/srep31819.

[32] J.D. Graci, C.E. Cameron, Mechanisms of action of ribavirin against distinct viruses, Rev. Med. Virol. 16 (2006) 37-48. doi:10.1002/rmv.483.

[33] K.M. Feibelman, B.P. Fuller, L. Li, D. V. LaBarbera, B.J. Geiss, Identification of small molecule inhibitors of the Chikungunya virus nsP1 RNA capping enzyme, Antiviral Res. 154 (2018) 124. doi:10.1016/J.ANTIVIRAL.2018.03.013.

[34] E. De Clercq, G. Li, Approved antiviral drugs over the past 50 years, Clin. Microbiol. Rev. 29 (2016) 695-747. doi:10.1128/CMR.00102-15.

[35] P.T. V Nguyen, H. Yu, P.A. Keller, Identification of chikungunya virus nsP2 protease inhibitors using structure-base approaches, J. Mol. Graph. Model. 57 (2015) 1-8. doi:10.1016/j.jmgm.2015.01.001.

[36] K.G. Byler, J.T. Collins, I.V. Ogungbe, W.N. Setzer, Alphavirus protease inhibitors from natural sources: A homology modeling and molecular docking investigation, Comput. Biol. Chem. 64 (2016) 163-184. doi:10.1016/j.compbiolchem.2016.06.005.

[37] P.K. Das, L. Puusepp, F.S. Varghese, A. Utt, T. Ahola, D.G. Kananovich, M. Lopp, A. Merits, M. Karelson, Design and validation of novel chikungunya virus protease inhibitors, Antimicrob. Agents Chemother. 7945 (2016) AAC.01421-16. doi:10.1128/AAC.01421-16. 
461

462

463

464

465

466

467

468

469

470

471

472

473

474

475

476

477

478

479

480

481

482

483

484

[38] S.S. Jadav, B.N. Sinha, R. Hilgenfeld, B. Pastorino, X. de Lamballerie, V. Jayaprakash, Thiazolidone derivatives as inhibitors of chikungunya virus, Eur. J. Med. Chem. 89 (2015) 172-178. doi:10.1016/j.ejmech.2014.10.042.

[39] E.M. El-Labbad, M. a H. Ismail, D. a Abou Ei Ella, M. Ahmed, F. Wang, K.H. Barakat, K. a M. Abouzid, Discovery of Novel Peptidomimetics as Irreversible CHIKV NsP2 Protease Inhibitors Using Quantum Mechanical-Based Ligand Descriptors., Chem. Biol. Drug Des. (2015) 1518-1527. doi:10.1111/cbdd.12621.

[40] L. Delang, N. Segura Guerrero, A. Tas, G. Quérat, B. Pastorino, M. Froeyen, K. Dallmeier, D. Jochmans, P. Herdewijn, F. Bello, E.J. Snijder, X. de Lamballerie, B. Martina, J. Neyts, M.J. van Hemert, P. Leyssen, Mutations in the chikungunya virus non-structural proteins cause resistance to favipiravir (T-705), a broad-spectrum antiviral., J. Antimicrob. Chemother. 69 (2014) 2770-84. doi:10.1093/jac/dku209.

[41] R. Abdelnabi, D. Jochmans, E. Verbeken, J. Neyts, L. Delang, Antiviral treatment efficiently inhibits chikungunya virus infection in the joints of mice during the acute but not during the chronic phase of the infection, Antiviral Res. (2017). doi:10.1016/j.antiviral.2017.09.016.

[42] M. Khan, R. Dhanwani, I.K. Patro, P.V.L. Rao, M.M. Parida, Cellular IMPDH enzyme activity is a potential target for the inhibition of Chikungunya virus replication and virus induced apoptosis in cultured mammalian cells, Antiviral Res. 89 (2011) 1-8. doi:10.1016/j.antiviral.2010.10.009.

[43] H. a. Rothan, H. Bahrani, Z. Mohamed, T.C. Teoh, E.M. Shankar, N. a. Rahman, R. Yusof, A Combination of Doxycycline and Ribavirin Alleviated Chikungunya Infection, PLoS One. 10 (2015) e0126360. doi:10.1371/journal.pone.0126360.

[44] S. Briolant, D. Garin, N. Scaramozzino, A. Jouan, J.M. Crance, In vitro inhibition of 
Chikungunya and Semliki Forest viruses replication by antiviral compounds: Synergistic effect of interferon-?? and ribavirin combination, Antiviral Res. 61 (2004) 111-117. doi:10.1016/j.antiviral.2003.09.005.

[45] P. Kaur, M. Thiruchelvan, R.C.H. Lee, H. Chen, K.C. Chen, M.L. Ng, J.J.H. Chu, Inhibition of Chikungunya virus replication by harringtonine, a novel antiviral that suppresses viral protein expression, Antimicrob. Agents Chemother. 57 (2013) 155-167. doi:10.1128/AAC.01467-12.

[46] S. Hover, B. Foster, J.N. Barr, J. Mankouri, Viral dependence on cellular ion channels an emerging antiviral target?, J. Gen. Virol. 98 (2017) 345-351. doi:10.1099/jgv.0.000712.

[47] M. Müller, N. Jones, E. Todd, H. Khalid, A. Merits, J. Mankouri, A. Tuplin, Replication of the Chikungunya virus genome requires cellular chloride channels, PLoS NegI Trop Dis. 13 (2019). doi:10.1099/acmi.ac2019.po0136.

[48] S. Ozden, M. Lucas-Hourani, P.E. Ceccaldi, A. Basak, M. Valentine, S. Benjannet, J. Hamelin, Y. Jacob, K. Mamchaoui, V. Mouly, P. Desprès, A. Gessain, G. Butler-Browne, M. Chrétien, F. Tangy, P.O. Vidalain, N.G. Seidah, Inhibition of Chikungunya virus infection in cultured human muscle cells by furin inhibitors: Impairment of the maturation of the E2 surface glycoprotein, J. Biol. Chem. 283 (2008) 21899-21908. doi:10.1074/jbc.M802444200.

[49] R. Broeckel, S. Sarkar, N.A. May, J. Totonchy, C.N. Kreklywich, P. Smith, L. Graves, V.R. DeFilippis, M.T. Heise, T.E. Morrison, N. Moorman, D.N. Streblow, Src Family Kinase Inhibitors Block Translation of Alphavirus Subgenomic mRNAs, Antimicrob. Agents Chemother. 63 (2019). doi:10.1128/aac.02325-18.

[50] F.S. Varghese, B. Thaa, S.N. Amrun, D. Simarmata, K. Rausalu, T.A. Nyman, A. Merits, 
G.M. Mclnerney, L.F.P. Ng, T. Ahola, The antiviral alkaloid berberine reduces chikungunya virus-induced mitogen-activated protein kinase (MAPK) signaling, J. Virol. (2016) JVI.01382-16. doi:10.1128/JVI.01382-16.

[51] R. Abdelnabi, S.N. Amrun, L.F.P. Ng, P. Leyssen, J. Neyts, L. Delang, Protein kinases C as potential host targets for the inhibition of chikungunya virus replication, Antiviral Res. 139 (2017) 79-87. doi:10.1016/j.antiviral.2016.12.020.

[52] R. Abdelnabi, D. Staveness, K.E. Near, P.A. Wender, L. Delang, J. Neyts, P. Leyssen, Comparative analysis of the anti-chikungunya virus activity of novel bryostatin analogs confirms the existence of a PKC-independent mechanism, Biochem. Pharmacol. 120 (2016) 15-21. doi:10.1016/j.bcp.2016.09.020.

[53] L.E. Cook, M.C. Locke, A.R. Young, K. Monte, M.L. Hedberg, R.M. Shimak, K.C.F. Sheehan, D.J. Veis, M.S. Diamond, D.J. Lenschow, Distinct roles of interferon alpha and beta in controlling chikungunya virus replication and modulating neutrophil-mediated inflammation, J. Virol. (2019). doi:10.1128/jvi.00841-19.

[54] K.M. Gallegos, G.L. Drusano, D.Z. D Argenio, A.N. Brown, Chikungunya Virus: In Vitro Response to Combination Therapy With Ribavirin and Interferon Alfa 2a., J. Infect. Dis. 214 (2016) 1192-7. doi:10.1093/infdis/jiw358.

[55] J.Y. Seo, R. Yaneva, P. Cresswell, Viperin: A multifunctional, interferon-inducible protein that regulates virus replication, Cell Host Microbe. 10 (2011) 534-539. doi:10.1016/j.chom.2011.11.004.

[56] Y. Zhang, C.W. Burke, K.D. Ryman, W.B. Klimstra, Identification and Characterization of Interferon-Induced Proteins That Inhibit Alphavirus Replication, J. Virol. 81 (2007) 11246-11255. doi:10.1128/jvi.01282-07.

[57] Y.G. Li, U. Siripanyaphinyo, U. Tumkosit, N. Noranate, A. A-Nuegoonpipat, Y. Pan, M. 
Kameoka, T. Kurosu, K. Ikuta, N. Takeda, S. Anantapreecha, Poly (I:C), an agonist of tolllike receptor-3, inhibits replication of the Chikungunya virus in BEAS-2B cells, Virol. J. 9 (2012). doi:10.1186/1743-422X-9-114.

[58] R. Priya, I.K. Patro, M.M. Parida, TLR3 mediated innate immune response in mice brain following infection with Chikungunya virus, Virus Res. 189 (2014) 194-205. doi:10.1016/j.virusres.2014.05.010.

[59] T. Matsumiya, D.M. Stafforini, Function and regulation of retinoic acid-inducible geneI, Crit. Rev. Immunol. 30 (2010) 489-513. doi:10.1615/critrevimmunol.v30.i6.10.

[60] M.L. Goulet, D. Olagnier, Z. Xu, S. Paz, S.M. Belgnaoui, E.I. Lafferty, V. Janelle, M. Arguello, M. Paquet, K. Ghneim, S. Richards, A. Smith, P. Wilkinson, M. Cameron, U. Kalinke, S. Qureshi, A. Lamarre, E.K. Haddad, R.P. Sekaly, S. Peri, S. Balachandran, R. Lin, J. Hiscott, Systems Analysis of a RIG-I Agonist Inducing Broad Spectrum Inhibition of Virus Infectivity, PLoS Pathog. 9 (2013). doi:10.1371/journal.ppat.1003298.

[61] D. Olagnier, F.E.M. Scholte, C. Chiang, I.C. Albulescu, C. Nichols, Z. He, R. Lin, E.J. Snijder, M.J. van Hemert, J. Hiscott, Inhibition of Dengue and Chikungunya Virus Infections by RIG-I-Mediated Type I Interferon-Independent Stimulation of the Innate Antiviral Response, J. Virol. 88 (2014) 4180-4194. doi:10.1128/jvi.03114-13.

[62] B. Gall, K. Pryke, J. Abraham, N. Mizuno, S. Botto, T.M. Sali, R. Broeckel, N. Haese, A. Nilsen, A. Placzek, T. Morrison, M. Heise, D. Streblow, V. DeFilippis, Emerging Alphaviruses Are Sensitive to Cellular States Induced by a Novel Small-Molecule Agonist of the STING Pathway., J. Virol. 92 (2018) e01913-17. doi:10.1128/JVI.01913-17.

[63] L.J. Herrero, S.-S. Foo, K.-C. Sheng, W. Chen, M.R. Forwood, R. Bucala, S. Mahalingam, Pentosan Polysulfate: a Novel Glycosaminoglycan-Like Molecule for Effective Treatment of Alphavirus-Induced Cartilage Destruction and Inflammatory Disease., J. 
Virol. 89 (2015) 8063-76. doi:10.1128/JVI.00224-15.

558

559

560

561

562

563

564

565

566

567

568

569

570

571

572

573

574

575

576

577

578

579

580

[64] A. Supramaniam, X. Liu, V. Ferro, L.J. Herrero, Prophylactic antiheparanase activity by PG545 is antiviral in vitro and protects against Ross River virus disease in mice, Antimicrob. Agents Chemother. 62 (2018). doi:10.1128/AAC.01959-17.

[65] L. Pohjala, A. Utt, M. Varjak, A. Lulla, A. Merits, T. Ahola, P. Tammela, Inhibitors of alphavirus entry and replication identified with a stable Chikungunya replicon cell line and virus-based assays, PLoS One. 6 (2011). doi:10.1371/journal.pone.0028923.

[66] A. Utt, P.K. Das, M. Varjak, V. Lulla, A. Lulla, A. Merits, Mutations Conferring a Noncytotoxic Phenotype on Chikungunya Virus Replicons Compromise Enzymatic Properties of Nonstructural Protein 2, J. Virol. 89 (2015) 3145-3162. doi:10.1128/jvi.03213-14.

[67] C. Weber, K. Sliva, C. von Rhein, B.M. Kümmerer, B.S. Schnierle, The green tea catechin, epigallocatechin gallate inhibits chikungunya virus infection, Antiviral Res. 113 (2015) 1-3. doi:10.1016/j.antiviral.2014.11.001.

[68] S. Selvarajah, N.R. Sexton, K.M. Kahle, R.H. Fong, K.A. Mattia, J. Gardner, K. Lu, N.M. Liss, B. Salvador, D.F. Tucker, T. Barnes, M. Mabila, X. Zhou, G. Rossini, J.B. Rucker, D.A. Sanders, A. Suhrbier, V. Sambri, A. Michault, M.O. Muench, B.J. Doranz, G. Simmons, A Neutralizing Monoclonal Antibody Targeting the Acid-Sensitive Region in Chikungunya Virus E2 Protects from Disease, PLoS Negl. Trop. Dis. 7 (2013). doi:10.1371/journal.pntd.0002423.

[69] S. Lam, K.C. Chen, M.M.L. Ng, J.J.H. Chu, Expression of Plasmid-Based shRNA against the E1 and nsP1 Genes Effectively Silenced Chikungunya Virus Replication, PLoS One. 7 (2012). doi:10.1371/journal.pone.0046396.

[70] A.P.S. Rathore, T. Haystead, P.K. Das, A. Merits, M.L. Ng, S.G. Vasudevan, Chikungunya 
virus nsP3 \& nsP4 interacts with HSP-90 to promote virus replication: HSP-90 inhibitors reduce CHIKV infection and inflammation in vivo, Antiviral Res. 103 (2014) 7-16. doi:10.1016/j.antiviral.2013.12.010.

584

585

586

587

588

589

590

591

592

593

594

595

596

597

598

599

600

601

602

603

604

[71] P. Kaur, J.J.H. Chu, Chikungunya virus: An update on antiviral development and challenges, Drug Discov. Today. 18 (2013) 969-983. doi:10.1016/j.drudis.2013.05.002.

[72] D.K. Gupta, P. Kaur, S.T. Leong, L.T. Tan, M.R. Prinsep, J.J.H. Chu, Anti-Chikungunya viral activities of aplysiatoxin-related compounds from the marine cyanobacterium Trichodesmium erythraeum, Mar. Drugs. $12 \quad$ (2014) 115-127. doi:10.3390/md12010115.

[73] R. Amorim, M.D.F. de Meneses, J.C. Borges, L.C. da Silva Pinheiro, L.A. Caldas, C.C. CirneSantos, M.V.P. de Mello, A.M.T. de Souza, H.C. Castro, I.C.N. de Palmer Paixão, R. de M. Campos, I.E. Bergmann, V. Malirat, A.M.R. Bernardino, M.A. Rebello, D.F. Ferreira, Thieno[2,3-b]pyridine derivatives: a new class of antiviral drugs against Mayaro virus, Arch. Virol. 162 (2017) 1577-1587. doi:10.1007/s00705-017-3261-0.

[74] F.C. Camini, T.F. da Silva, C.C. da Silva Caetano, L.T. Almeida, A.C. Ferraz, V.M. Alves Vitoreti, B. de Mello Silva, S. de Queiroz Silva, J.C. de Magalhães, C.L. de Brito Magalhães, Antiviral activity of silymarin against Mayaro virus and protective effect in virus-induced oxidative stress, Antiviral Res. 158 (2018) 8-12. doi:10.1016/j.antiviral.2018.07.023.

[75] D.J.M. Cruz, R.M. Bonotto, R.G.B. Gomes, C.T. da Silva, J.B. Taniguchi, J.H. No, B. Lombardot, O. Schwartz, M. a E. Hansen, L.H. Freitas-Junior, Identification of Novel Compounds Inhibiting Chikungunya Virus-Induced Cell Death by High Throughput Screening of a Kinase Inhibitor Library, PLoS Negl. Trop. Dis. 7 (2013). doi:10.1371/journal.pntd.0002471. 
605 [76] X. Li, H. Zhang, Y. Zhang, J. Li, Z. Wang, C. Deng, A.C.G. Jardim, A.C.B. Terzian, M.L.

606

607

608

609

610

611

612

613

614

615

616

617

618

619

620

621

622

623

624

625

626

627

628

Nogueira, B. Zhang, Development of a rapid antiviral screening assay based on eGFP reporter virus of Mayaro virus, Antiviral Res. 168 (2019) 82-90. doi:10.1016/J.ANTIVIRAL.2019.05.013.

[77] F.S. Varghese, P. Kaukinen, S. Gläsker, M. Bespalov, L. Hanski, K. Wennerberg, A. Kummerer, Beate. Merits, T. Ahola, Discovery of berberine, abamectin and ivermectin as antivirals against chikungunya and other alphaviruses, Antiviral Res. 126 (2016) 117124. doi:10.1016/j.antiviral.2015.12.012.

[78] D.F. Smee, B.L. Hurst, W.J. Evans, N. Clyde, S. Wright, C. Peterson, K.-H. Jung, C.W. Day, Evaluation of cell viability dyes in antiviral assays with RNA viruses that exhibit different cytopathogenic properties., J. Virol. Methods. $246 \quad$ (2017) 51-57. doi:10.1016/j.jviromet.2017.03.012.

[79] A. Gómez-Sanjuan, A.M. Gamo, L. Delang, A. Pérez-Sánchez, S.N. Amrun, R. Abdelnabi, S. Jacobs, E.M. Priego, M.J. Camarasa, D. Jochmans, P. Leyssen, L.F.P. Ng, G. Querat, J. Neyts, M.J. Pérez-Pérez, Inhibition of the Replication of Different Strains of Chikungunya Virus by 3-Aryl-[1,2,3]triazolo[4,5- d] pyrimidin-7(6 H)-ones, ACS Infect. Dis. 4 (2018) 605-619. doi:10.1021/acsinfecdis.7b00219.

[80] A. Suhrbier, M.-C. Jaffar-Bandjee, P. Gasque, Arthritogenic alphaviruses-an overview, (2012). doi:10.1038/nrrheum.2012.64.

[81] E.Y. Gong, J.-F. Bonfanti, T. Ivens, M. Van der Auwera, B. Van Kerckhove, G. Kraus, Development of a High-Throughput Antiviral Assay for Screening Inhibitors of Chikungunya Virus and Generation of Drug-Resistant Mutations in Cultured Cells, in: Humana Press, Totowa, NJ, 2013: pp. 429-438. doi:10.1007/978-1-62703-484-5_32.

[82] G.C. Roberts, C. Zothner, R. Remenyi, A. Merits, N.J. Stonehouse, M. Harris, Evaluation 
of a range of mammalian and mosquito cell lines for use in Chikungunya virus research., Sci. Rep. 7 (2017) 14641. doi:10.1038/s41598-017-15269-w.

[83] S. Chen, Q. Zhang, X. Wu, P.G. Schultz, S. Ding, Dedifferentiation of Lineage-Committed Cells by a Small Molecule, (2003). doi:10.1021/JA037390K.

[84] S. He, P. Jain, B. Lin, M. Ferrer, Z. Hu, N. Southall, X. Hu, W. Zheng, B. Neuenswander, C.-H. Cho, Y. Chen, S.A. Worlikar, J. Aubé, R.C. Larock, F.J. Schoenen, J.J. Marugan, T.J. Liang, K.J. Frankowski, High-Throughput Screening, Discovery, and Optimization To Develop a Benzofuran Class of Hepatitis C Virus Inhibitors., ACS Comb. Sci. 17 (2015) 641-52. doi:10.1021/acscombsci.5b00101.

[85] T.E. Morrison, L. Oko, S. a. Montgomery, A.C. Whitmore, A.R. Lotstein, B.M. Gunn, S. a. Elmore, M.T. Heise, A mouse model of chikungunya virus-induced musculoskeletal inflammatory disease: Evidence of arthritis, tenosynovitis, myositis, and persistence, Am. J. Pathol. 178 (2011) 32-40. doi:10.1016/j.ajpath.2010.11.018.

[86] N.E. Rulli, M.S. Rolph, A. Srikiatkhachorn, S. Anantapreecha, A. Guglielmotti, S. Mahalingam, Protection From Arthritis and Myositis in a Mouse Model of Acute Chikungunya Virus Disease by Bindarit, an Inhibitor of Monocyte Chemotactic Protein1 Synthesis, J. Infect. Dis. 204 (2011) 1026-1030. doi:10.1093/infdis/jir470.

[87] T.E. Morrison, A.C. Whitmore, R.S. Shabman, B.A. Lidbury, S. Mahalingam, M.T. Heise, Characterization of Ross River virus tropism and virus-induced inflammation in a mouse model of viral arthritis and myositis., J. Virol. $80 \quad$ (2006) 737-49. doi:10.1128/JVI.80.2.737-749.2006.

[88] B.A. Lidbury, N.E. Rulli, A. Suhrbier, P.N. Smith, S.R. McColl, A.L. Cunningham, A. Tarkowski, N. van Rooijen, R.J. Fraser, S. Mahalingam, Macrophage-Derived Proinflammatory Factors Contribute to the Development of Arthritis and Myositis after 
653

654

655

656

657

658

659

660

661

662

663

664

665

666

667

668

669

670

671

672

673

674

675

676

Infection with an Arthrogenic Alphavirus, J. Infect. Dis. 197 (2008) 1585-1593. doi:10.1086/587841.

[89] L.J. Herrero, B.A. Lidbury, J. Bettadapura, P. Jian, B.L. Herring, W.J. Hey-Cunningham, K.-C. Sheng, A. Zakhary, S. Mahalingam, Characterization of Barmah Forest virus pathogenesis in a mouse model, J. Gen. Virol. 95 (2014) 2146-2154. doi:10.1099/vir.0.064733-0.

[90] C. Figueiredo, R. Neris, D. Leopoldino, J. Almeida, J. dos-Santos, C. Figueiredo, M. Bellio, M. Bozza, I. Assunção-Miranda, Mayaro virus replication restriction and induction of muscular inflammation in mice are dependent on age and type-l interferon response, bioRxiv. (2019) 602920. doi:10.1101/602920.

[91] B.A. Lidbury, C. Simeonovic, G.E. Maxwell, I.D. Marshall, A.J. Hapel, MacrophageInduced Muscle Pathology Results in Morbidity and Mortality for Ross River VirusInfected Mice, J. Infect. Dis. 181 (2000) 27-34. doi:10.1086/315164.

[92] F.M. Santos, R.S. Dias, M.D. de Oliveira, I.C.T.A. Costa, L. de S. Fernandes, C.R. Pessoa, S.L.P. da Matta, V.V. Costa, D.G. Souza, C.C. da Silva, S.O. de Paula, Animal model of arthritis and myositis induced by the Mayaro virus, PLoS Negl. Trop. Dis. 13 (2019) e0007375. doi:10.1371/journal.pntd.0007375.

[93] J. Gardner, I. Anraku, T.T. Le, T. Larcher, L. Major, P. Roques, W.A. Schroder, S. Higgs, A. Suhrbier, Chikungunya virus arthritis in adult wild-type mice., J. Virol. 84 (2010) 802132. doi:10.1128/JVI.02603-09.

[94] R.L. Seymour, S.L. Rossi, N.A. Bergren, K.S. Plante, S.C. Weaver, The role of innate versus adaptive immune responses in a mouse model of O'nyong-nyong virus infection., Am. J. Trop. Med. Hyg. 88 (2013) 1170-9. doi:10.4269/ajtmh.12-0674.

[95] T.A. Smith-Norowitz, R.A. Sobel, F. Mokhtarian, B Cells and Antibodies in the 
Pathogenesis of Myelin Injury in Semliki Forest Virus Encephalomyelitis, Cell. Immunol. 200 (2000) 27-35. doi:10.1006/CIMM.2000.1613.

[96] D.C. Thach, T. Kimura, D.E. Griffin, Differences between C57BL/6 and BALB/cBy mice in mortality and virus replication after intranasal infection with neuroadapted Sindbis virus., J. Virol. 74 (2000) 6156-61. doi:10.1128/jvi.74.13.6156-6161.2000.

[97] S.H. Cook, D.E. Griffin, Luciferase Imaging of a Neurotropic Viral Infection in Intact Animals, J. Virol. 77 (2003) 5333-5338. doi:10.1128/JVI.77.9.5333-5338.2003.

[98] K. Pesko, C.J. Westbrook, C.N. Mores, L.P. Lounibos, M.H. Reiskind, Effects of Infectious Virus Dose and Bloodmeal Delivery Method on Susceptibility of Aedes aegypti and Aedes albopictus to Chikungunya Virus, J. Med. Entomol. 46 (2009) 395-399. doi:10.1603/033.046.0228.

[99] D. Sissoko, D. Malvy, K. Ezzedine, P. Renault, F. Moscetti, M. Ledrans, V. Pierre, Postepidemic Chikungunya disease on reunion island: Course of rheumatic manifestations and associated factors over a 15-month period, PLoS Negl. Trop. Dis. 3 (2009). doi:10.1371/journal.pntd.0000389.

[100] H. Salje, J. Lessler, K.K. Paul, A.S. Azman, M.W. Rahman, M. Rahman, D. Cummings, E.S. Gurley, S. Cauchemez, How social structures, space, and behaviors shape the spread of infectious diseases using chikungunya as a case study, Proc. Natl. Acad. Sci. 113 (2016) 201611391. doi:10.1073/pnas.1611391113. 